\title{
New Possibility of Direct Observations of Quantum Dots, Nanotubes and Interfaces by Cs-Corrected HRTEM
}

\author{
N. Tanaka, J. Yamasaki, H. Sawada ${ }^{\#}$ and M. Naruse ${ }^{\#}$ \\ C.I.R.S.E., Nagoya University, Nagoya, 464-8603, Japan \\ ${ }^{\#}$ EOD, JEOL Ltd., Akishima, Tokyo, 196-8558, Japan
}

Recently various types of technologies for correction of spherical aberration are realized[1-3]. Although problems still remain in the damping envelope functions due to energy-spread of incident beam and various kinds of electrical and mechanical instabilities, the effectiveness of spherical aberration correction(Cs-correction) should be now qualified in high-resolution TEM/STEM images of various nano-materials. We have developed a new Cs-corrected TEM based on a 200kV HRTEM with FEG (JEM-2010F) as shown in Fig.1[4] and report here its characteristics for observation of quantum dots, nanotubes and interfaces.

The technology of Cs-correction in objective lens of TEM was established by Rose and Haider group[1]. Two hexapoles and two transfer lenses were used for the correction. The assessment/correction of lens-coefficients was performed using the well-known Zemlin tableaus caused by incident beam-tilt up to $18 \mathrm{mrad}$. The high-resolution images were recorded by a digital CCD camera with pixels of $1 \mathrm{k} \times 1 \mathrm{k}$. The samples were (1)InGaAs quantum dots prepared by OMVPE, (2)carbon nanotubes, (3) $\mathrm{SiGe} / \mathrm{Si}$ interfaces prepared by chemical vapor deposition and (4) $\mathrm{SiO}_{2} / \mathrm{Si}$ interfaces[5].

Fig. 2 shows an image of a part of InGaAs quantum dot embedded in GaP. The dumbbell-image of $\mathrm{Ga}$ and $\mathrm{P}$ atomic columns is almost resolved as indicated by two arrows, but that in the InGaAs area is deteriorated into (111) lattice fringes, which is due to a local tilt of the area caused by lattice-strain. Careful comparison between actual images and simulated ones gives the local tilt quantitatively[6]. Fig. 3 shows a Cs-corrected TEM image of a carbon nanotube. Since the nanotube is an ideal phase-object or weak phase object, the image contrast is to be none with $\mathrm{Cs}=0$ and $\Delta \mathrm{f}=0$ as indicated by an arrow. The phenomenon is used for detection of small height-difference in nano-materials. Fig. 4 shows the similar image of a (111) staking fault of $\mathrm{SiGe}$ alloy[7]. Due to no Fresnel fringe contrast, the image contrast is apparently weak, but directly interpretable. The extrinsic nature of the fault was clarified by comparison between the image and simulated ones. With the optimization of the FEG and electrical and mechanical instabilities, the microscope may be able to attain $0.12 \mathrm{~nm}$ in point-to-point resolution even in a $200 \mathrm{kV}$ instrument.

In the present study, we have proved other characteristics for Cs-corrected TEM such as (1)clear observation of lattice-fringe contrast at the zero-defocus[4] and (2)accurately selected-area diffraction, etc. Although these are well-known in principle, but now we have been able to actually experience those in the present Cs-corrected TEM. We have a plan to use the instrument with a new possibility for dynamical observations, whose techniques were established in our laboratory for small systems such as single atoms, clusters and nano-wires[8]. 
References

[1] M.Haider et al., J. Electron Microsc. 47 (1998) 395.

[2] O.L Krivanek et al., Ultramicrosc. 78 (1999) 1.

[3] Y. Takai et al., Proc. $14^{\text {th }}$ I.C.E.M. (1998) Vol.1 115.

[4] N. Tanaka et al., Proc. $14^{\text {th }}$ I.C.E.M. (2002) Vol.3 37.

[5] N. Tanaka et al., J. Electron Microsc. 52(2003) No.2, in print.

[6] N. Tanaka et al., Microscopy and Microanalysis (2003), to be submitted.

[7] N. Tanaka et al., Proc. E.M.A.G. (2002) p.373.

[8] N. Tanaka \& T. Kizuka, Bull. of MRS 22(1997) 58.

[9] The authors would like to thank Drs. M.Haider and P.Hartel of CEOS GmbH for the present experiments, and Prof. Y.Ikuhara of Tokyo University, Dr. T.Hirayama of Japan Fine Ceramics Center, Prof. Y.Takeda of Nagoya University and Messrs. T.Tomita and F.Hosokawa of JEOL Co for their kind support and sample-preparation. The present study is partly supported by a Special Grant as "Active-Nano Characterization", a Grant-in-Aid for a Scientific Research Project of "Highly Functionized Global Interface Integration" from the Ministry of Education, Culture, Sports, Science and Technology and a CREST Grant for "New Development of Quantum dots"(\#47-004) from Japan Science and Technology Corporation.

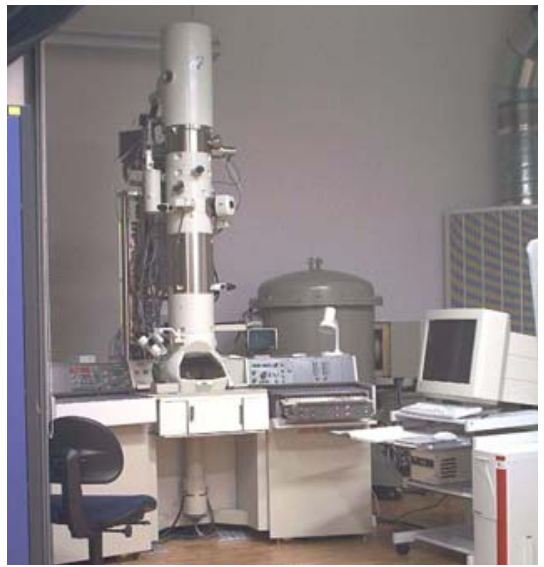

Fig.1: Photo of a new Cs-corrected TEM based on JEM-2010F.

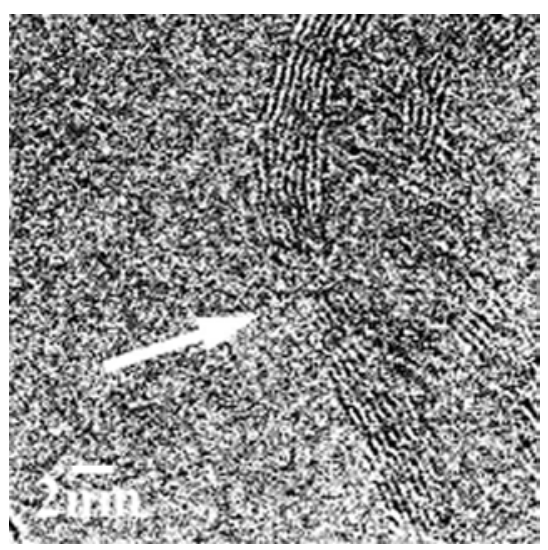

Fig. 3: Disappearance of lattice fringes in a carbon nanotube.

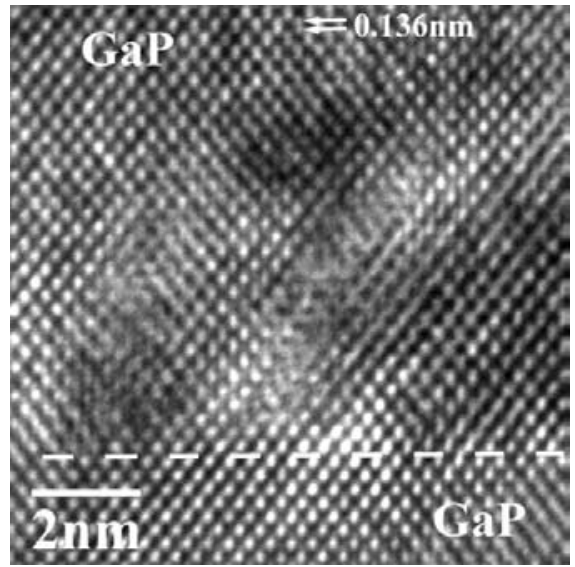

Fig. 2: Cs-corrected HRETM image of an InGaAs quantum dot in GaP.

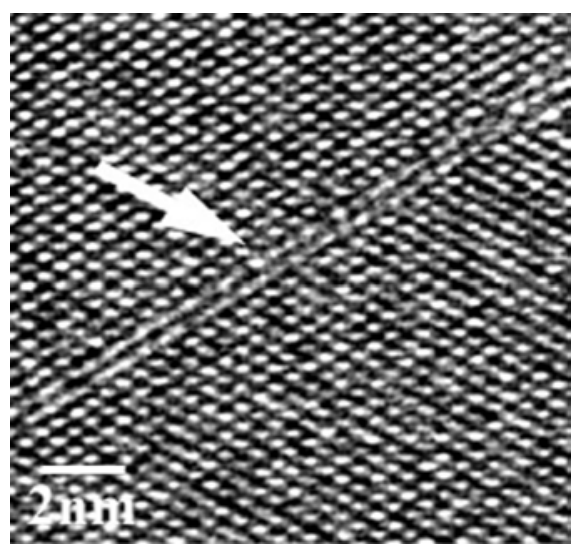

Fig. 4: Cs-corrected HRTEM image of a stacking fault in a SiGe alloy. 